\title{
Primary pulmonary lymphoma presenting as nodules and masses: a case report and literature review
}

\author{
Xiaofang Gao, Peng Yan \\ Department of Pulmonary Medicine, Chinese People's Liberation Army General Hospital, Beijing, China
}

\begin{abstract}
Primary pulmonary lymphoma (PPL) is a rare neoplasm. We report a case of a 45-year-old man who was admitted to hospital for an abnormal CT scan found incidentally in annual health screening. The contrast-enhanced chest CT scans revealed multiple nodules and masses of varying sizes in right upper and middle lobes and both lower lobes. CT-guided percutaneous transthoracic needle biopsy was performed. The diagnosis of PPL was confirmed by histopathological examination and Immunohistochemical staining. PPL should be included in the differential diagnoses in symptomless patients with multiple pulmonary nodules and masses.
\end{abstract}

Correspondence: Dr. Xiaofang Gao, Department of Pulmonary Medicine, Chinese People's Liberation Army General Hospital, 28 Fuxing Road, Beijing 100853, China.

Mobile: +86.15810759096 .

E-mail: gaoxiaofang1977@163.com.

Key words: Primary pulmonary lymphoma; computed tomography; diagnosis.

Contributions: All the authors contributed equally. All the authors have read and approved the final version of the manuscript and agreed to be accountable for all aspects of the work.

Funding: No funding support was taken for the conduct of the study.

Conflict of interest: The Authors declare no conflict of interest.

Ethics approval and consent to participate: No ethical committee approval was required for this case report by the Department, because this article does not contain any studies with human participants or animals.

Consent for publication: The patient gave his written consent to use his personal data for the publication of this case report and any accompanying images.

Received for publication: 29 February 2020.

Accepted for publication: 26 November 2020.

${ }^{\circ}$ Copyright: the Author(s), 2021

Licensee PAGEPress, Italy

Monaldi Archives for Chest Disease 2021; 91:1259

doi: 10.4081/monaldi.2021.1259

This article is distributed under the terms of the Creative Commons Attribution Noncommercial License (by-nc 4.0) which permits any noncommercial use, distribution, and reproduction in any medium, provided the original author(s) and source are credited.

\section{Introduction}

Primary pulmonary lymphoma (PPL) is a rare disease and can present with non-specific symptoms [1]. The radiological presentation of PPL is heterogeneous, presenting as consolidation, pleural effusion, patchy infiltration, masses and nodules [2]. These characteristics can often pose diagnostic challenges to clinicians and delay the diagnosis. Here we report a case of PPL presenting with multiple pulmonary nodules and masses.

\section{Case Report}

A 45-year-old man who worked in a coal mine was admitted to the hospital for evaluation of multiple pulmonary masses. Two months ago, multiple masses in both lungs were incidentally found by chest computed tomography (CT) scans in annual health screening, and fine needle puncture of the lesion in right lower lobe guided by CT was carried out in local hospital. Histopathological examination showed fibrous connective tissue infiltrated by inflammatory cells, and the patients received prednisone $(30 \mathrm{mg} /$ day) for 5 weeks for the suspicious diagnosis of pulmonary sarcoidosis.

After admission, the contrast enhanced chest CT scan showed multiple nodules and masses of varying sizes in right upper and middle lobes and both lower lobes (Figure 1), and there were no obvious changes compared with two months ago. Cerebral magnetic resonance imaging (MRI) and abdominal ultrasonography were normal. Neither mediastinal lymph node enlargement nor lymphadenopathy at any other site was present. Clinically the patient was asymptomatic and there were no abnormal physical signs. The complete blood count, erythrocyte sedimentation rate (ESR), serum angiotensin converting enzyme (ACE) and serum tumor markers were within normal limits.

A further CT-guided percutaneous transthoracic needle biopsy was performed for the mass in the right upper lobe. The histologic examination of biopsy specimen showed diffuse infiltrates of small to medium-size lymphoid cells. Immunohistochemical staining demonstrated that the cells were positive for Bcl-2, CD3, CD20, CD21, CD43 and Kappa, and they were negative for CD10, Cyclin D1, TTF-1 and Lambda; The Ki-67 labeling index was $25-50 \%$. Based on these findings, the diagnosis of PPL (low-grade extranodal marginal zone B-cell lymphoma) was made. The patient was referred to department of Hematology-Oncology and received combination chemotherapy (cyclophosphamide, adriamycin, oncovin and prednisone; CHOP). 


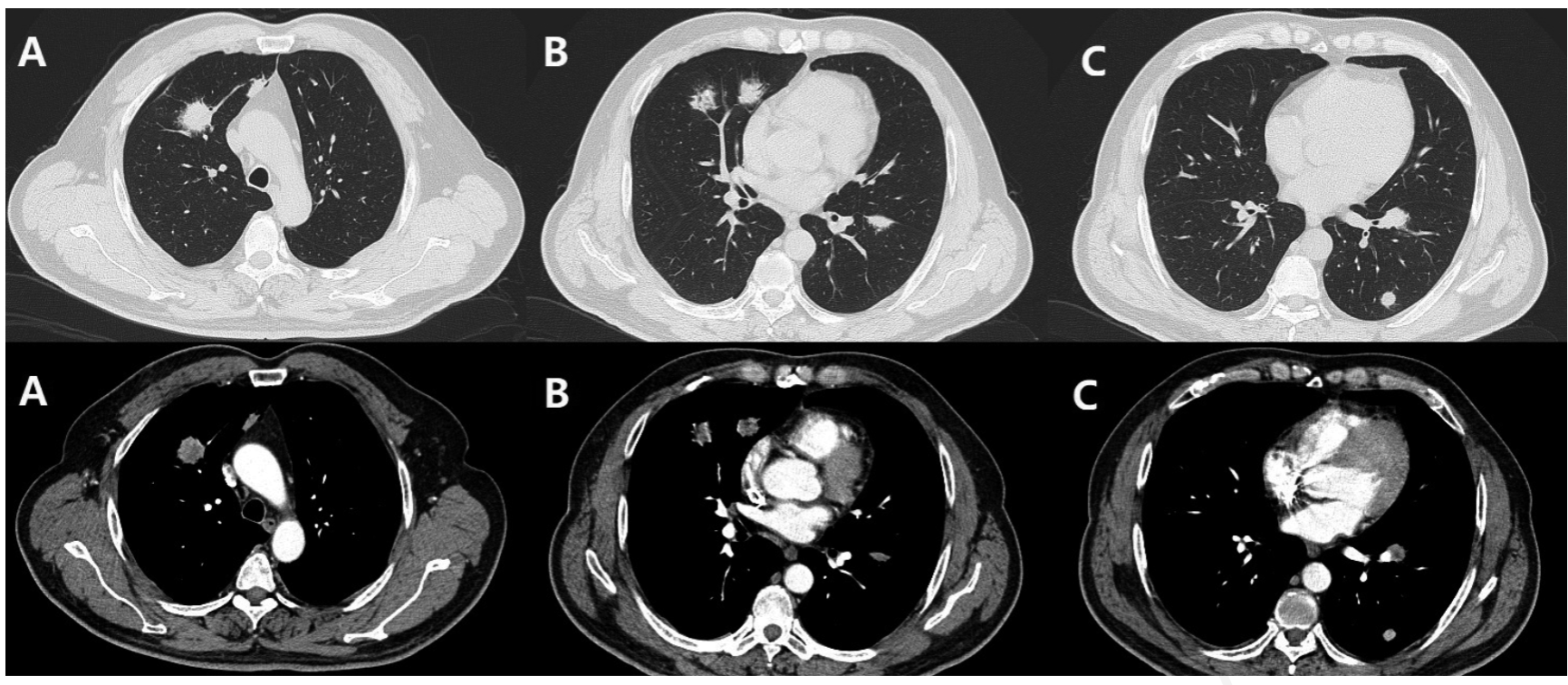

Figure 1. Contrast enhanced CT of chest on admission reveals variable sized nodules and masses in right upper lobe (A), right middle lobe (B), and left lower lobe (C). There is no enlarged mediastinal lymph node.

\section{Discussion}

PPL is a rare disorder, representing only $0.5-1 \%$ of all primary pulmonary malignancies, less than $1 \%$ of all cases of non-Hodgkin's lymphoma (NHL) and 3-4\% of all extranodal manifestations of NHL [3]. PPL is defined as clonal lymphoid proliferation affecting one or both lungs in a patient with no detectable extrapulmonary involvement at diagnosis or during the subsequent 3 months [4]. According to the World Health Organization's classification system, PPL can be divided into two types: mucosa-associated lymphoid tissue (MALT) and non-MALT. The former is represented by the lowgrade extranodal marginal zone B-cell lymphoma (58\%-87\%), while the latter consists of diffuse large B-cell or high-grade lymphoma (11\%-19\%) and anaplastic large cell lymphoma [5].

Primary pulmonary MALT lymphoma often occurs in the fifth to seventh decade of life, and individuals under the age of 30 years are rarely affected [6]. Most studies report that the two genders are equally affected. Nearly half of the patients are asymptomatic at diagnosis, incidentally identified solely on the basis of a radiological pulmonary anomaly. When presenting, symptoms like cough, mild dyspnoea or chest pain are nonspecific. Extrapulmonary manifestations are limited to general signs (fever, malaise or weight loss) and occur in less than one quarter of patients [7].

The radiographic appearance of PPL showed a variety of findings, such as single or multiple nodules, masses, ill-defined infiltrates and consolidations [8]. This suggests that any radiological abnormality of the lung parenchyma contains the possibility of lymphoma. Positron emission tomography (PET) scan is of certain clinical value for evaluation of the lung lesions, but its imaging is nonspecific. As illustrated by this case, the diagnosis of PPL rests on pathologic findings, thus further emphasizing the importance of prompt transbronchial or transthoracic needle biopsy in suspected cases. Immunohistochemical analysis contributes to the differential and positive diagnosis of MALT-type PPL [9]. The interval between the first clinical or radiological manifestation and diagnosis ranges from 5 months to 8 years.
Several treatment options are available, including tumor resection, radiotherapy, chemotherapy and combined therapy [10]. Exclusive chemotherapy is generally used for patients with bilateral or extrapulmonary involvement, relapse or progression. The prognosis of PPL is generally excellent in most cases, with a 5-year survival rate of over $80 \%$ and a median survival time of over 10 years [11]

\section{Conclusions}

The diagnosis of PPL is challenging and typically dependent on immunohistochemical analysis of biopsy specimens. PPL should be included in the differential diagnoses in symptomless patients with multiple pulmonary nodules and masses.

\section{References}

1. Yao D, Zhang L, Wu PL, et al. Clinical and misdiagnosed analysis of primary pulmonary lymphoma: a retrospective study. BMC Cancer 2018;18:281.

2. Bai Y, Liang W. CT and PET/CT findings of primary pulmonary diffuse large B-cell lymphoma: One case report and literature review. Medicine (Baltimore) 2017;96:e8876.

3. Shin CH, Paik SH, Park JS, et al. Primary pulmonary T-cell lymphoma: a case report. Korean J Radiol 2010;11:234-8.

4. Cadranel J, Wislez M, Antoine M. Primary pulmonary lymphoma. Eur Respir J 2002;20:750-62.

5. Cardenas-Garcia J, Talwar A, Shah R, Fein A. Update in primary pulmonary lymphomas. Curr Opin Pulm Med 2015;21:333-7.

6. Kocatürk Cİ, Seyhan EC, Günlüoğlu MZ, et al. Primary pulmonary non-Hodgkin's lymphoma: ten cases with a review of the literature. Tuberk Toraks 2012;60:246-53.

7. Tillawi IS. Primary pulmonary Hodgkin's lymphoma. A report 
of 2 cases and review of the literature. Saudi Med J 2007;28:943-8.

8. Qian J, Luo DL, Zhang JE, et al. Diagnostic and prognostic factors for patients with primary pulmonary non-Hodgkin's lymphoma: A 16-year single-center retrospective study. Oncol Lett;18:2082-90.

9. Lee J, Park H, Kim YW, Yoo CG. Pulmonary Lymphoma
Misdiagnosed as Pneumonia. Indian J Hematol Blood Transfus 2016;32:509-11.

10. Wannesson L, Cavalli F, Zucca E. Primary pulmonary lymphoma: current status. Clin Lymphoma Myeloma 2005;6:220-7.

11. Cooksley N, Judge DJ, Brown J. Primary pulmonary Hodgkin's lymphoma and a review of the literature since 2006. BMJ Case Rep 2014;2014:bcr2014204020. 